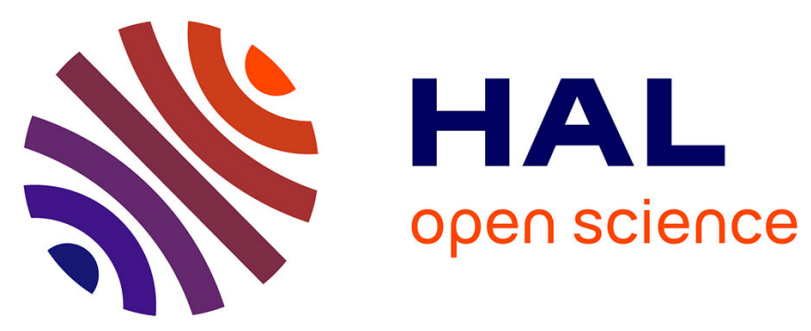

\title{
Solvability in the sense of sequences for some non Fredholm operators related to the superdiffusion
}

\author{
Vitali Vougalter, Vitaly Volpert
}

\section{To cite this version:}

Vitali Vougalter, Vitaly Volpert. Solvability in the sense of sequences for some non Fredholm operators related to the superdiffusion. Journal of Pseudo-Differential Operators and Applications, 2017, 160, pp.1-22. 10.1007/s11868-017-0204-1 . hal-01573772

\section{HAL Id: hal-01573772 \\ https://hal.science/hal-01573772}

Submitted on 10 Aug 2017

HAL is a multi-disciplinary open access archive for the deposit and dissemination of scientific research documents, whether they are published or not. The documents may come from teaching and research institutions in France or abroad, or from public or private research centers.
L'archive ouverte pluridisciplinaire HAL, est destinée au dépôt et à la diffusion de documents scientifiques de niveau recherche, publiés ou non, émanant des établissements d'enseignement et de recherche français ou étrangers, des laboratoires publics ou privés. 


\title{
SOLVABILITY IN THE SENSE OF SEQUENCES FOR SOME NON FREDHOLM OPERATORS RELATED TO THE SUPERDIFFUSION
}

\author{
Vitali Vougalter ${ }^{1}$, Vitaly Volpert ${ }^{2,3}$ \\ ${ }^{1}$ Department of Mathematics, University of Toronto \\ Toronto, Ontario, M5S 2E4, Canada \\ e-mail: vitali@math.toronto.edu \\ ${ }^{2}$ Institute Camille Jordan, UMR 5208 CNRS, University Lyon 1 \\ Villeurbanne, 69622, France \\ ${ }^{3}$ RUDN University, ul. Miklukho-Maklaya 6, Moscow, 117198, Russia \\ e-mail: volpert@math.univ-lyon1.fr
}

\begin{abstract}
We study solvability of some linear nonhomogeneous elliptic equations and show that under reasonable technical conditions the convergence in $L^{2}\left(\mathbb{R}^{d}\right)$ of their right sides yields the existence and the convergence in $H^{1}\left(\mathbb{R}^{d}\right)$ of the solutions. The problems involve the square roots of the second order non Fredholm differential operators and we use the methods of spectral and scattering theory for Schrödinger type operators similarly to our preceding work [28].
\end{abstract}

AMS Subject Classification: 35J10, 35P10, 47F05

Key words: solvability conditions, non Fredholm operators, Sobolev spaces

\section{Introduction}

Let us consider the problem

$$
\sqrt{-\Delta+V(x)} u-a u=f
$$

with $u \in E=H^{1}\left(\mathbb{R}^{d}\right)$ and $f \in F=L^{2}\left(\mathbb{R}^{d}\right), d \in \mathbb{N}, a$ is a constant and $V(x)$ is a function decaying to 0 at infinity. The operator $\sqrt{-\Delta+V(x)}$ can be defined via the spectral calculus under the appropriate technical conditions on the scalar potential $V(x)$ (see Assumption 3 below). If $a \geq 0$, then the essential spectrum of the operator $A: E \rightarrow F$ corresponding to the left side of problem (1.1) contains the origin. Consequently, such operator fails to satisfy the Fredholm property. Its image is not closed, for $d>1$ the dimensions of its kernel and the codimension of its image are not finite. In the present article we will study some properties of 
the operators of this kind. Let us recall that elliptic equations with non-Fredholm operators were treated extensively in recent years (see [18], [19], [20], [21], [22], [23], [25], [26], also [5]) along with their potential applications to the theory of reaction-diffusion equations (see [7], [8]). In the particular case when $a=0$ the operator $A^{2}$ satisfies the Fredholm property in some properly chosen weighted spaces [1], [2], [3], [4], [5]. However, the case with $a \neq 0$ is significantly different and the approach developed in these articles cannot be adopted.

One of the important questions concerning problems with non-Fredholm operators is their solvability. Let us address it in the following setting. Let $f_{n}$ be a sequence of functions in the image of the operator $A$, such that $f_{n} \rightarrow f$ in $L^{2}\left(\mathbb{R}^{d}\right)$ as $n \rightarrow \infty$. Denote by $u_{n}$ a sequence of functions from $H^{1}\left(\mathbb{R}^{d}\right)$ such that

$$
A u_{n}=f_{n}, n \in \mathbb{N} .
$$

Because the operator $A$ fails to satisfy the Fredholm property, the sequence $u_{n}$ may not be convergent. Let us call a sequence $u_{n}$ such that $A u_{n} \rightarrow f$ a solution in the sense of sequences of problem $A u=f$ (see [17]). If such sequence converges to a function $u_{0}$ in the norm of the space $E$, then $u_{0}$ is a solution of this problem. Solution in the sense of sequences is equivalent in this sense to the usual solution. However, in the case of the operators without Fredholm property, this convergence may not hold or it can occur in some weaker sense. In such case, solution in the sense of sequences may not imply the existence of the usual solution. In the present article we will find sufficient conditions of equivalence of solutions in the sense of sequences and the usual solutions. In the other words, the conditions on sequences $f_{n}$ under which the corresponding sequences $u_{n}$ are strongly convergent. Solvability in the sense of sequences for the sums of non Fredholm Schrödinger type operators was treated in [27].

In the first part of the work we consider the problem

$$
\sqrt{-\Delta} u-a u=f(x), x \in \mathbb{R}^{d}, d \in \mathbb{N}
$$

where $a \geq 0$ is a constant and the right side is square integrable. The operator $\sqrt{-\Delta}$ here is actively used, for instance in the studies of the superdiffusion problems (see e.g. [29] and the references therein). Superdiffusion can be described as a random process of particle motion characterized by the probability density distribution of jump length. The moments of this density distribution are finite in the case of normal diffusion, but this is not the case for superdiffusion. Asymptotic behavior at infinity of the probability density function determines the value of the power of the Laplacian (see [14]). Another application of the $\sqrt{-\Delta}$ operator is related to the relativistic Quantum Mechanics (see e.g. [24]). The equation analogous to (1.2) but with the standard Laplacian in the context of the solvability in the sense of sequences was treated in [28]. Note that for the operator $\sqrt{-\Delta}-a: H^{1}\left(\mathbb{R}^{d}\right) \rightarrow L^{2}\left(\mathbb{R}^{d}\right)$ the essential spectrum fills the semi-axis $[-a, \infty)$ such that its inverse from $L^{2}\left(\mathbb{R}^{d}\right)$ to $H^{1}\left(\mathbb{R}^{d}\right)$ is unbounded. 
We write down the corresponding sequence of equations with $n \in \mathbb{N}$ as

$$
\sqrt{-\Delta} u_{n}-a u_{n}=f_{n}(x), x \in \mathbb{R}^{d}, d \in \mathbb{N}
$$

with the right sides convergent to the right side of (1.2) in $L^{2}\left(\mathbb{R}^{d}\right)$ as $n \rightarrow \infty$. The inner product of two functions

$$
(f(x), g(x))_{L^{2}\left(\mathbb{R}^{d}\right)}:=\int_{\mathbb{R}^{d}} f(x) \bar{g}(x) d x
$$

with a slight abuse of notations when these functions fail to be square integrable. Indeed, if $f(x) \in L^{1}\left(\mathbb{R}^{d}\right)$ and $g(x) \in L^{\infty}\left(\mathbb{R}^{d}\right)$, then obviously the integral in the right side of (1.4) makes sense, like for example in the case of functions involved in the orthogonality conditions of Theorems 1 and 2 below. We will use the space $H^{1}\left(\mathbb{R}^{d}\right)$ equipped with the norm

$$
\|u\|_{H^{1}\left(\mathbb{R}^{d}\right)}^{2}:=\|u\|_{L^{2}\left(\mathbb{R}^{d}\right)}^{2}+\|\nabla u\|_{L^{2}\left(\mathbb{R}^{d}\right)}^{2} .
$$

Throughout the article, the sphere of radius $r>0$ in $\mathbb{R}^{d}$ centered at the origin will be denoted by $S_{r}^{d}$. The notation $B^{d}$ will stand for the unit ball in the space of $d$ dimensions with the center at the origin and $\left|B^{d}\right|$ for its Lebesgue measure. First of all, let us formulate the solvability conditions for equation (1.2).

Theorem 1. Let $f(x) \in L^{2}\left(\mathbb{R}^{d}\right), d \in \mathbb{N}$.

a) When $a=0, d=1,2$ and in addition $x f(x) \in L^{1}\left(\mathbb{R}^{d}\right)$, problem (1.2) admits a unique solution $u(x) \in H^{1}\left(\mathbb{R}^{d}\right)$ if and only if

$$
(f(x), 1)_{L^{2}\left(\mathbb{R}^{d}\right)}=0
$$

holds.

b) When $a=0, d \geq 3$ and in addition $f(x) \in L^{1}\left(\mathbb{R}^{d}\right)$, problem (1.2) possesses a unique solution $u(x) \in H^{1}\left(\mathbb{R}^{d}\right)$.

c) When $a>0, d=1$ and in addition $x f(x) \in L^{1}(\mathbb{R})$, equation (1.2) has a unique solution $u(x) \in H^{1}(\mathbb{R})$ if and only if

$$
\left(f(x), \frac{e^{ \pm i a x}}{\sqrt{2 \pi}}\right)_{L^{2}(\mathbb{R})}=0
$$

holds.

d) When $a>0, d \geq 2$ and in addition $x f(x) \in L^{1}\left(\mathbb{R}^{d}\right)$, problem (1.2) admits a unique solution $u(x) \in H^{1}\left(\mathbb{R}^{d}\right)$ if and only if

$$
\left(f(x), \frac{e^{i p x}}{(2 \pi)^{\frac{d}{2}}}\right)_{L^{2}\left(\mathbb{R}^{d}\right)}=0, \quad p \in S_{a}^{d}
$$


holds.

Then let us turn our attention to the issue of the solvability in the sense of sequences for our equation.

Theorem 2. Let $n \in \mathbb{N}$ and $f_{n}(x) \in L^{2}\left(\mathbb{R}^{d}\right), d \in \mathbb{N}$, such that $f_{n}(x) \rightarrow f(x)$ in $L^{2}\left(\mathbb{R}^{d}\right)$ as $n \rightarrow \infty$.

a) When $a=0, d=1,2$, let in addition $x f_{n}(x) \in L^{1}\left(\mathbb{R}^{d}\right), n \in \mathbb{N}$, such that $x f_{n}(x) \rightarrow x f(x)$ in $L^{1}\left(\mathbb{R}^{d}\right)$ as $n \rightarrow \infty$ and the orthogonality conditions

$$
\left(f_{n}(x), 1\right)_{L^{2}\left(\mathbb{R}^{d}\right)}=0
$$

hold for all $n \in \mathbb{N}$. Then problems (1.2) and (1.3) admit unique solutions $u(x) \in$ $H^{1}\left(\mathbb{R}^{d}\right)$ and $u_{n}(x) \in H^{1}\left(\mathbb{R}^{d}\right)$ respectively, such that $u_{n}(x) \rightarrow u(x)$ in $H^{1}\left(\mathbb{R}^{d}\right)$ as $n \rightarrow \infty$.

b) When $a=0, d \geq 3$, let in addition $f_{n}(x) \in L^{1}\left(\mathbb{R}^{d}\right), n \in \mathbb{N}$, such that $f_{n}(x) \rightarrow f(x)$ in $L^{1}\left(\mathbb{R}^{d}\right)$ as $n \rightarrow \infty$. Then equations (1.2) and (1.3) possess unique solutions $u(x) \in H^{1}\left(\mathbb{R}^{d}\right)$ and $u_{n}(x) \in H^{1}\left(\mathbb{R}^{d}\right)$ respectively, such that $u_{n}(x) \rightarrow$ $u(x)$ in $H^{1}\left(\mathbb{R}^{d}\right)$ as $n \rightarrow \infty$.

c) When $a>0, d=1$, let in addition $x f_{n}(x) \in L^{1}(\mathbb{R}), n \in \mathbb{N}$, such that $x f_{n}(x) \rightarrow x f(x)$ in $L^{1}(\mathbb{R})$ as $n \rightarrow \infty$ and the orthogonality conditions

$$
\left(f_{n}(x), \frac{e^{ \pm i a x}}{\sqrt{2 \pi}}\right)_{L^{2}(\mathbb{R})}=0
$$

hold for all $n \in \mathbb{N}$. Then equations (1.2) and (1.3) have unique solutions $u(x) \in$ $H^{1}(\mathbb{R})$ and $u_{n}(x) \in H^{1}(\mathbb{R})$ respectively, such that $u_{n}(x) \rightarrow u(x)$ in $H^{1}(\mathbb{R})$ as $n \rightarrow \infty$.

d) When $a>0, d \geq 2$, let in addition $x f_{n}(x) \in L^{1}\left(\mathbb{R}^{d}\right), n \in \mathbb{N}$, such that $x f_{n}(x) \rightarrow x f(x)$ in $L^{1}\left(\mathbb{R}^{d}\right)$ as $n \rightarrow \infty$ and the orthogonality conditions

$$
\left(f_{n}(x), \frac{e^{i p x}}{(2 \pi)^{\frac{d}{2}}}\right)_{L^{2}\left(\mathbb{R}^{d}\right)}=0, \quad p \in S_{a}^{d}
$$

hold for all $n \in \mathbb{N}$. Then equations (1.2) and (1.3) admit unique solutions $u(x) \in$ $H^{1}\left(\mathbb{R}^{d}\right)$ and $u_{n}(x) \in H^{1}\left(\mathbb{R}^{d}\right)$ respectively, such that $u_{n}(x) \rightarrow u(x)$ in $H^{1}\left(\mathbb{R}^{d}\right)$ as $n \rightarrow \infty$.

Remark. In the statement b) of Theorem 2 our operator A has a right inverse. However, since $L^{1}\left(\mathbb{R}^{d}\right) \cap L^{2}\left(\mathbb{R}^{d}\right)$ is not a Hilbert space, the classical theory of operators acting in Hilbert spaces (see e.g. [10]) is not applicable here. The inverse image of the operator may not be closed in $H^{1}\left(\mathbb{R}^{d}\right)$. Similar assertions can be made for analogous statements of the other theorems. On the other hand, 
similarly to the Laplace operator (see e.g. [1]- [5]), it is possible that the operator under consideration satisfies the Fredholm property in the appropriate weighted spaces. This is not yet proved for the fractional operators.

Note that when $a=0$ and the dimension of the problem is at least three, orthogonality relations in the theorems above are not required.

Let us use the hat symbol to designate the standard Fourier transform

$$
\widehat{f}(p):=\frac{1}{(2 \pi)^{\frac{d}{2}}} \int_{\mathbb{R}^{d}} f(x) e^{-i p x} d x, p \in \mathbb{R}^{d}, d \in \mathbb{N}
$$

such that

$$
\|\widehat{f}(p)\|_{L^{\infty}\left(\mathbb{R}^{d}\right)} \leq \frac{1}{(2 \pi)^{\frac{d}{2}}}\|f(x)\|_{L^{1}\left(\mathbb{R}^{d}\right)} .
$$

In the second part of the article we treat the problem

$$
\sqrt{-\Delta+V(x)} u-a u=f(x), x \in \mathbb{R}^{3}, a \geq 0,
$$

where the right side is square integrable. The correspondent sequence of equations for $n \in \mathbb{N}$ will be

$$
\sqrt{-\Delta+V(x)} u_{n}-a u_{n}=f_{n}(x), x \in \mathbb{R}^{3}, a \geq 0,
$$

where the right sides converge to the right side of (1.14) in $L^{2}\left(\mathbb{R}^{3}\right)$ as $n \rightarrow \infty$. We make the following technical assumptions on the scalar potential involved in equations above. Note that the conditions on $V(x)$, which is shallow and shortrange will be analogous to those stated in Assumption 1.1 of [19] (see also [20], [21]). The essential spectrum of such a Schrödinger operator fills the nonnegative semi-axis (see e.g. [11]).

Assumption 3. The potential function $V(x): \mathbb{R}^{3} \rightarrow \mathbb{R}$ satisfies the estimate

$$
|V(x)| \leq \frac{C}{1+|x|^{3.5+\delta}}
$$

with some $\delta>0$ and $x=\left(x_{1}, x_{2}, x_{3}\right) \in \mathbb{R}^{3}$ a.e. such that

$$
4^{\frac{1}{9}} \frac{9}{8}(4 \pi)^{-\frac{2}{3}}\|V\|_{L^{\infty}\left(\mathbb{R}^{3}\right)}^{\frac{1}{9}}\|V\|_{L^{\frac{4}{3}\left(\mathbb{R}^{3}\right)}}^{\frac{8}{9}}<1 \quad \text { and } \quad \sqrt{c_{H L S}}\|V\|_{L^{\frac{3}{2}\left(\mathbb{R}^{3}\right)}}<4 \pi \text {. }
$$

Here and below $C$ will denote a finite positive constant and $c_{H L S}$ given on p.98 of [13] is the constant in the Hardy-Littlewood-Sobolev inequality

$$
\left|\int_{\mathbb{R}^{3}} \int_{\mathbb{R}^{3}} \frac{f_{1}(x) f_{1}(y)}{|x-y|^{2}} d x d y\right| \leq c_{H L S}\left\|f_{1}\right\|_{L^{\frac{3}{2}\left(\mathbb{R}^{3}\right)}}^{2}, \quad f_{1} \in L^{\frac{3}{2}}\left(\mathbb{R}^{3}\right) .
$$


By means of Lemma 2.3 of [19], under Assumption 3 above on the potential function, the operator $-\Delta+V(x)$ on $L^{2}\left(\mathbb{R}^{3}\right)$ is self-adjoint and unitarily equivalent to $-\Delta$ via the wave operators (see [12], [16])

$$
\Omega^{ \pm}:=s-\lim _{t \rightarrow \mp \infty} e^{i t(-\Delta+V)} e^{i t \Delta},
$$

where the limit is understood in the strong $L^{2}$ sense (see e.g. [15] p.34, [6] p.90). Thus $\sqrt{-\Delta+V(x)}: H^{1}\left(\mathbb{R}^{3}\right) \rightarrow L^{2}\left(\mathbb{R}^{3}\right)$ has only the essential spectrum

$$
\sigma_{e s s}(\sqrt{-\Delta+V(x)}-a)=[-a, \infty)
$$

and no nontrivial $L^{2}\left(\mathbb{R}^{3}\right)$ eigenfunctions. Its functions of the continuous spectrum satisfy

$$
\sqrt{-\Delta+V(x)} \varphi_{k}(x)=|k| \varphi_{k}(x), \quad k \in \mathbb{R}^{3},
$$

in the integral formulation the Lippmann-Schwinger equation for the perturbed plane waves (see e.g. [15] p.98)

$$
\varphi_{k}(x)=\frac{e^{i k x}}{(2 \pi)^{\frac{3}{2}}}-\frac{1}{4 \pi} \int_{\mathbb{R}^{3}} \frac{e^{i|k||x-y|}}{|x-y|}\left(V \varphi_{k}\right)(y) d y
$$

and the orthogonality relations

$$
\left(\varphi_{k}(x), \varphi_{q}(x)\right)_{L^{2}\left(\mathbb{R}^{3}\right)}=\delta(k-q), \quad k, q \in \mathbb{R}^{3} .
$$

In particular, when the vector $k=0$, we have $\varphi_{0}(x)$. We designate the generalized Fourier transform with respect to these functions using the tilde symbol as

$$
\tilde{f}(k):=\left(f(x), \varphi_{k}(x)\right)_{L^{2}\left(\mathbb{R}^{3}\right)}, k \in \mathbb{R}^{3} .
$$

(1.20) is a unitary transform on $L^{2}\left(\mathbb{R}^{3}\right)$. The integral operator involved in (1.18) is being designated as

$$
(Q \varphi)(x):=-\frac{1}{4 \pi} \int_{\mathbb{R}^{3}} \frac{e^{i|k||x-y|}}{|x-y|}(V \varphi)(y) d y, \quad \varphi \in L^{\infty}\left(\mathbb{R}^{3}\right) .
$$

We consider $Q: L^{\infty}\left(\mathbb{R}^{3}\right) \rightarrow L^{\infty}\left(\mathbb{R}^{3}\right)$. Under Assumption 3, according to Lemma 2.1 of [19] the operator norm $\|Q\|_{\infty}$ is bounded above by the quantity $I(V)$, which is the left side of the first inequality in (1.16), such that $I(V)<1$. Corollary 2.2 of [19] under our assumptions gives us the estimate

$$
|\tilde{f}(k)| \leq \frac{1}{(2 \pi)^{\frac{3}{2}}} \frac{1}{1-I(V)}\|f(x)\|_{L^{1}\left(\mathbb{R}^{3}\right)} .
$$

We have the following statement concerning the solvability of problem (1.14).

Theorem 4. Let Assumption 3 hold and $f(x) \in L^{2}\left(\mathbb{R}^{3}\right)$. 
a) When $a=0$, let in addition $f(x) \in L^{1}\left(\mathbb{R}^{3}\right)$. Then equation (1.14) admits a unique solution $u(x) \in H^{1}\left(\mathbb{R}^{3}\right)$.

b) When $a>0$, let in addition $x f(x) \in L^{1}\left(\mathbb{R}^{3}\right)$. Then equation (1.14) has a unique solution $u(x) \in H^{1}\left(\mathbb{R}^{3}\right)$ if and only if

$$
\left(f(x), \varphi_{k}(x)\right)_{L^{2}\left(\mathbb{R}^{3}\right)}=0, \quad k \in S_{a}^{3}
$$

holds.

Our final main proposition deals with the solvability in the sense of sequences of equation (1.14).

Theorem 5. Let Assumption 3 hold, $n \in \mathbb{N}$ and $f_{n}(x) \in L^{2}\left(\mathbb{R}^{3}\right)$, such that $f_{n}(x) \rightarrow f(x)$ in $L^{2}\left(\mathbb{R}^{3}\right)$ as $n \rightarrow \infty$.

a) When $a=0$ let in addition $f_{n}(x) \in L^{1}\left(\mathbb{R}^{3}\right), n \in \mathbb{N}$, such that $f_{n}(x) \rightarrow f(x)$ in $L^{1}\left(\mathbb{R}^{3}\right)$ as $n \rightarrow \infty$. Then problems (1.14) and (1.15) possess unique solutions $u(x) \in H^{1}\left(\mathbb{R}^{3}\right)$ and $u_{n}(x) \in H^{1}\left(\mathbb{R}^{3}\right)$ respectively, such that $u_{n}(x) \rightarrow u(x)$ in $H^{1}\left(\mathbb{R}^{3}\right)$ as $n \rightarrow \infty$.

b) When $a>0$ let in addition $x f_{n}(x) \in L^{1}\left(\mathbb{R}^{3}\right), n \in \mathbb{N}$, such that $x f_{n}(x) \rightarrow$ $x f(x)$ in $L^{1}\left(\mathbb{R}^{3}\right)$ as $n \rightarrow \infty$ and the orthogonality conditions

$$
\left(f_{n}(x), \varphi_{k}(x)\right)_{L^{2}\left(\mathbb{R}^{3}\right)}=0, k \in S_{a}^{3}
$$

hold for all $n \in \mathbb{N}$. Then problems (1.14) and (1.15) possess unique solutions $u(x) \in H^{1}\left(\mathbb{R}^{3}\right)$ and $u_{n}(x) \in H^{1}\left(\mathbb{R}^{3}\right)$ respectively, such that $u_{n}(x) \rightarrow u(x)$ in $H^{1}\left(\mathbb{R}^{3}\right)$ as $n \rightarrow \infty$.

Note that (1.22) and (1.23) are the orthogonality conditions to the functions of the continuous spectrum of our Schrödinger operator, as distinct from the Limiting Absorption Principle in which one needs to orthogonalize to the standard Fourier harmonics (see e.g. Lemma 2.3 and Proposition 2.4 of [9]).

\section{Solvability in the sense of sequences in the no potential case}

Proof of Theorem 1. Note that the statements a) and b) of the theorem are the results of Lemma 3.1 of [29].

Evidently, if $u(x) \in L^{2}\left(\mathbb{R}^{d}\right)$ is a solution of (1.2) with a square integrable right side, it belongs to $H^{1}\left(\mathbb{R}^{d}\right)$ as well. Indeed, directly from (1.2) we have $\sqrt{-\Delta} u(x) \in$ $L^{2}\left(\mathbb{R}^{d}\right)$, such that due to the fact that

$$
\|\sqrt{-\Delta} u\|_{L^{2}\left(\mathbb{R}^{d}\right)}=\|\nabla u\|_{L^{2}\left(\mathbb{R}^{d}\right)}
$$

we have $\nabla u(x) \in L^{2}\left(\mathbb{R}^{d}\right)$, which yields $u(x) \in H^{1}\left(\mathbb{R}^{d}\right)$ as well. 
To show the uniqueness of solutions for our problem, let us suppose that (1.2) admits two square integrable solutions $u_{1}(x)$ and $u_{2}(x)$. Then their difference $w(x):=u_{1}(x)-u_{2}(x) \in L^{2}\left(\mathbb{R}^{d}\right)$ as well. Clearly, it satisfies the equation

$$
\sqrt{-\Delta} w=a w .
$$

Since the operator $\sqrt{-\Delta}$ has no nontrivial square integrable eigenfunctions in the whole space, we have $w(x)=0$ a.e. in $\mathbb{R}^{d}$.

Let us apply the standard Fourier transform (1.12) to both sides of equation (1.2). This yields

$$
\widehat{u}(p)=\frac{\widehat{f}(p)}{|p|-a} .
$$

Let us first treat the case c) of the theorem, such that the dimension of the problem $d=1$. We define the following sets on the real line

$$
I_{\delta}^{+}:=[a-\delta, a+\delta], \quad I_{\delta}^{-}:=[-a-\delta,-a+\delta], \quad 0<\delta<a,
$$

such that

$$
I_{\delta}:=I_{\delta}^{+} \cup I_{\delta}^{-}, \quad \mathbb{R}=I_{\delta} \cup I_{\delta}^{c} .
$$

Here and further down $A^{c} \subseteq \mathbb{R}^{d}$ denotes the complement of the set $A \subseteq \mathbb{R}^{d}$. This enables us to express $\widehat{u}(p)$ as the sum

$$
\frac{\widehat{f}(p)}{|p|-a} \chi_{I_{\delta}^{c}}+\frac{\widehat{f}(p)}{|p|-a} \chi_{I_{\delta}^{+}}+\frac{\widehat{f}(p)}{|p|-a} \chi_{I_{\delta}^{-}} .
$$

Note that $\chi_{I}$ stands for the characteristic function of an interval $I$ on the real line. Obviously, we have $I_{\delta}^{c}=I_{\delta}^{c+} \cup I_{\delta}^{c-}$, where

$$
I_{\delta}^{c+}:=I_{\delta}^{c} \cap \mathbb{R}^{+}, \quad I_{\delta}^{c-}:=I_{\delta}^{c} \cap \mathbb{R}^{-} .
$$

Here $\mathbb{R}^{+}$and $\mathbb{R}^{-}$are the nonnegative and the negative semi-axes of the real line respectively. Apparently,

$$
\left|\frac{\widehat{f}(p)}{p-a} \chi_{I_{\delta}^{c+}}\right| \leq \frac{|\widehat{f}(p)|}{\delta} \in L^{2}(\mathbb{R}) .
$$

Similarly,

$$
\left|\frac{\widehat{f}(p)}{-p-a} \chi_{I_{\delta}^{c-}}\right| \leq \frac{|\widehat{f}(p)|}{\delta} \in L^{2}(\mathbb{R}) .
$$

Let us use the representation

$$
\widehat{f}(p)=\widehat{f}(a)+\int_{a}^{p} \frac{d \widehat{f}(s)}{d s} d s .
$$


From the definition (1.12) of the Fourier transform we easily obtain the bound by the finite quantity due to the one of our assumptions, namely

$$
\left|\frac{d \widehat{f}(p)}{d p}\right| \leq \frac{1}{\sqrt{2 \pi}}\|x f(x)\|_{L^{1}(\mathbb{R})}
$$

This easily yields the upper bound

$$
\left|\frac{\int_{a}^{p} \frac{d \widehat{f}(s)}{d s} d s}{p-a} \chi_{I_{\delta}^{+}}\right| \leq \frac{1}{\sqrt{2 \pi}}\|x f(x)\|_{L^{1}(\mathbb{R})} \chi_{I_{\delta}^{+}} \in L^{2}(\mathbb{R}) .
$$

Apparently,

$$
\frac{\widehat{f}(a)}{p-a} \chi_{I_{\delta}^{+}} \in L^{2}(\mathbb{R})
$$

if and only if $\widehat{f}(a)=0$, which is equivalent to the orthogonality condition

$$
\left(f(x), \frac{e^{i a x}}{\sqrt{2 \pi}}\right)_{L^{2}(\mathbb{R})}=0 .
$$

To treat the singularity of the problem on the negative semi-axis, we use the formula

$$
\widehat{f}(p)=\widehat{f}(-a)+\int_{-a}^{p} \frac{d \widehat{f}(s)}{d s} d s .
$$

This gives us the upper bound

$$
\left|\frac{\int_{-a}^{p} \frac{d \widehat{f}(s)}{d s} d s}{-p-a} \chi_{I_{\delta}^{-}}\right| \leq \frac{1}{\sqrt{2 \pi}}\|x f(x)\|_{L^{1}(\mathbb{R})} \chi_{I_{\delta}^{-}} \in L^{2}(\mathbb{R}) .
$$

Evidently,

$$
\frac{\widehat{f}(-a)}{-p-a} \chi_{I_{\delta}^{-}} \in L^{2}(\mathbb{R})
$$

if and only if $\widehat{f}(-a)=0$, which is equivalent to the orthogonality relation

$$
\left(f(x), \frac{e^{-i a x}}{\sqrt{2 \pi}}\right)_{L^{2}(\mathbb{R})}=0 .
$$

Let us complete the proof of the theorem with establishing the part d). When the dimension $d \geq 2$, we introduce the set

$$
A_{\delta}:=\left\{p \in \mathbb{R}^{d}|a-\delta \leq| p \mid \leq a+\delta\right\}, \quad 0<\delta<a
$$


and express

$$
\widehat{u}(p)=\frac{\widehat{f}(p)}{|p|-a} \chi_{A_{\delta}}+\frac{\widehat{f}(p)}{|p|-a} \chi_{A_{\delta}^{c}} .
$$

Clearly, we have the upper bound

$$
\left|\frac{\widehat{f}(p)}{|p|-a} \chi_{A_{\delta}^{c}}\right| \leq \frac{|\widehat{f}(p)|}{\delta} \in L^{2}\left(\mathbb{R}^{d}\right) .
$$

To study the first term in the right side of (2.27), we will use the representation formula

$$
\widehat{f}(p)=\widehat{f}(a, \sigma)+\int_{a}^{|p|} \frac{\partial \widehat{f}(s, \sigma)}{\partial s} d s .
$$

Here and further down $\sigma$ will stand for the angle variables on the sphere. By means for the definition of the Fourier transform (1.12), we have the upper bound

$$
\left|\frac{\partial \widehat{f}(p)}{\partial|p|}\right| \leq \frac{\|x f(x)\|_{L^{1}\left(\mathbb{R}^{d}\right)}}{(2 \pi)^{\frac{d}{2}}} .
$$

The right side of this inequality is finite due to the one of our assumptions. This allows us to estimate

$$
\left|\frac{\int_{a}^{|p|} \frac{\partial \widehat{f}(s, \sigma)}{\partial s} d s}{|p|-a} \chi_{A_{\delta}}\right| \leq \frac{\|x f(x)\|_{L^{1}\left(\mathbb{R}^{d}\right)}}{(2 \pi)^{\frac{d}{2}}} \chi_{A_{\delta}} \in L^{2}\left(\mathbb{R}^{d}\right) .
$$

The remaining term

$$
\frac{\widehat{f}(a, \sigma)}{|p|-a} \chi_{A_{\delta}} \in L^{2}\left(\mathbb{R}^{d}\right)
$$

if and only if $\widehat{f}(a, \sigma)$ vanishes, which is equivalent to orthogonality condition (1.8) for the dimensions $d \geq 2$.

Then we turn our attention to establishing the solvability in the sense of sequences for our problem in the no potential case.

Proof of Theorem 2. Suppose $u(x)$ and $u_{n}(x), n \in \mathbb{N}$ are the unique solutions of equations (1.2) and (1.3) in $H^{1}\left(\mathbb{R}^{d}\right), d \in \mathbb{N}$ with $a \geq 0$ respectively and it is known that $u_{n}(x) \rightarrow u(x)$ in $L^{2}\left(\mathbb{R}^{d}\right)$ as $n \rightarrow \infty$. Then $u_{n}(x) \rightarrow u(x)$ in $H^{1}\left(\mathbb{R}^{d}\right)$ as $n \rightarrow \infty$ as well. Indeed,

$$
\sqrt{-\Delta}\left(u_{n}(x)-u(x)\right)=a\left(u_{n}(x)-u(x)\right)+\left(f_{n}(x)-f(x)\right),
$$

which clearly yields

$$
\left\|\sqrt{-\Delta}\left(u_{n}(x)-u(x)\right)\right\|_{L^{2}\left(\mathbb{R}^{d}\right)} \leq a\left\|u_{n}(x)-u(x)\right\|_{L^{2}\left(\mathbb{R}^{d}\right)}+\left\|f_{n}(x)-f(x)\right\|_{L^{2}\left(\mathbb{R}^{d}\right)} \rightarrow 0
$$


as $n \rightarrow \infty$ due to our assumptions. It can be easily verified that the left side of the inequality above equals to

$$
\left\|\nabla\left(u_{n}(x)-u(x)\right)\right\|_{L^{2}\left(\mathbb{R}^{d}\right)} \rightarrow 0, \quad n \rightarrow \infty .
$$

If $u(x)$ and $u_{n}(x), n \in \mathbb{N}$ are the unique solutions of problems (1.2) and (1.3) in $H^{1}\left(\mathbb{R}^{d}\right), d \in \mathbb{N}$ respectively with $a=0$ as in the cases a) and b) of the theorem, by applying the standard Fourier transform (1.12) we easily obtain

$$
\widehat{u}_{n}(p)-\widehat{u}(p)=\frac{\widehat{f}_{n}(p)-\widehat{f}(p)}{|p|} \chi_{\{|p| \leq 1\}}+\frac{\widehat{f}_{n}(p)-\widehat{f}(p)}{|p|} \chi_{\{|p|>1\}} .
$$

Clearly, the second term in the right side of identity (2.28) can be bounded from above in the absolute value by $\left|\widehat{f}_{n}(p)-\widehat{f}(p)\right|$, such that

$$
\left\|\frac{\widehat{f}_{n}(p)-\widehat{f}(p)}{|p|} \chi_{\{|p|>1\}}\right\|_{L^{2}\left(\mathbb{R}^{d}\right)} \leq\left\|f_{n}(x)-f(x)\right\|_{L^{2}\left(\mathbb{R}^{d}\right)} \rightarrow 0, \quad n \rightarrow \infty
$$

by means of the one of our assumptions. Let us first address the case a) of the theorem when the dimension $d=1$. Then via the part a) of Theorem 1 , each equation (1.3) admits a unique solution $u_{n}(x) \in H^{1}(\mathbb{R}), \quad n \in \mathbb{N}$. Apparently,

$$
\left|(f(x), 1)_{L^{2}(\mathbb{R})}\right|=\left|\left(f(x)-f_{n}(x), 1\right)_{L^{2}(\mathbb{R})}\right| \leq\left\|f_{n}(x)-f(x)\right\|_{L^{1}(\mathbb{R})} \rightarrow 0
$$

as $n \rightarrow \infty$ due to the result of Lemma 6 below. Hence,

$$
(f(x), 1)_{L^{2}(\mathbb{R})}=0
$$

holds. By virtue of the part a) of Theorem 1, equation (1.2) has a unique solution $u(x) \in H^{1}(\mathbb{R})$ when the dimension $d=1$ and $a=0$. Orthogonality conditions (2.29) and (1.9) give us

$$
\widehat{f}(0)=0, \quad \widehat{f}_{n}(0)=0, \quad n \in \mathbb{N}
$$

in such case. This enables us to use the representations

$$
\widehat{f}(p)=\int_{0}^{p} \frac{d \widehat{f}(s)}{d s} d s, \quad \widehat{f}_{n}(p)=\int_{0}^{p} \frac{d \widehat{f}_{n}(s)}{d s} d s, \quad n \in \mathbb{N}
$$

which allows us to write the first term in the right side of identity (2.28) as

$$
\frac{\int_{0}^{p}\left(\frac{d \widehat{f}_{n}(s)}{d s}-\frac{d \widehat{f}(s)}{d s}\right) d s}{|p|} \chi_{\{|p| \leq 1\}}
$$


Using definition (1.12) of the standard Fourier transform, we easily estimate

$$
\left|\frac{d \widehat{f}_{n}(p)}{d p}-\frac{d \widehat{f}(p)}{d p}\right| \leq \frac{1}{\sqrt{2 \pi}}\left\|x f_{n}(x)-x f(x)\right\|_{L^{1}(\mathbb{R})},
$$

such that expression (2.30) can be bounded from above in the absolute value by

$$
\frac{1}{\sqrt{2 \pi}}\left\|x f_{n}(x)-x f(x)\right\|_{L^{1}(\mathbb{R})} \chi_{\{|p| \leq 1\}}
$$

Therefore,

$$
\left\|\frac{\int_{0}^{p}\left(\frac{d \widehat{f}_{n}(s)}{d s}-\frac{d \widehat{f}(s)}{d s}\right) d s}{|p|} \chi_{\{|p| \leq 1\}}\right\|_{L^{2}(\mathbb{R})} \leq \frac{1}{\sqrt{\pi}}\left\|x f_{n}(x)-x f(x)\right\|_{L^{1}(\mathbb{R})} \rightarrow 0
$$

as $n \rightarrow \infty$ due to the one of our assumptions. This implies that

$$
u_{n}(x) \rightarrow u(x) \quad \text { in } \quad L^{2}(\mathbb{R}), \quad n \rightarrow \infty
$$

when the dimension $d=1$ and $a=0$. In the case of the dimension $d=2$ and $a=0$, orthogonality condition

$$
(f(x), 1)_{L^{2}\left(\mathbb{R}^{2}\right)}=0
$$

can be obtained via the trivial limiting argument, analogously to (2.29). By means of the part a) of Theorem 1, equations (1.2) and (1.3) admit unique solutions $u(x) \in$ $H^{1}\left(\mathbb{R}^{2}\right)$ and $u_{n}(x) \in H^{1}\left(\mathbb{R}^{2}\right), n \in \mathbb{N}$ respectively. Orthogonality relations (2.32) and (1.9) yield

$$
\widehat{f}(0)=0, \quad \widehat{f}_{n}(0)=0, \quad n \in \mathbb{N}
$$

when the dimension $d=2$ and $a=0$ as well. This allows us to express

$$
\widehat{f}(p)=\int_{0}^{|p|} \frac{\partial \widehat{f}(s, \sigma)}{\partial s} d s, \quad \widehat{f}_{n}(p)=\int_{0}^{|p|} \frac{\partial \widehat{f}_{n}(s, \sigma)}{\partial s} d s, \quad n \in \mathbb{N} .
$$

Then the first term in the right side of identity (2.28) can be written as

$$
\frac{\int_{0}^{|p|}\left(\frac{\partial \widehat{f}_{n}(s, \sigma)}{\partial s}-\frac{\partial \widehat{f}(s, \sigma)}{\partial s}\right) d s}{|p|} \chi_{\{|p| \leq 1\}} .
$$

By means of definition (1.12) of the standard Fourier transform, we easily arrive at

$$
\left|\frac{\partial \widehat{f}_{n}(p)}{\partial|p|}-\frac{\partial \widehat{f}(p)}{\partial|p|}\right| \leq \frac{1}{2 \pi}\left\|x f_{n}(x)-x f(x)\right\|_{L^{1}\left(\mathbb{R}^{2}\right)}
$$


Hence, expression (2.33) can be estimated from above in the absolute value by

$$
\frac{1}{2 \pi}\left\|x f_{n}(x)-x f(x)\right\|_{L^{1}\left(\mathbb{R}^{2}\right)} \chi_{\{|p| \leq 1\}},
$$

such that

$$
\left\|\frac{\int_{0}^{|p|}\left(\frac{\partial \widehat{f}_{n}(s, \sigma)}{\partial s}-\frac{\partial \widehat{f}(s, \sigma)}{\partial s}\right) d s}{|p|} \chi_{\{|p| \leq 1\}}\right\|_{L^{2}\left(\mathbb{R}^{2}\right)} \leq \frac{1}{2 \sqrt{\pi}}\left\|x f_{n}(x)-x f(x)\right\|_{L^{1}\left(\mathbb{R}^{2}\right)} \rightarrow 0
$$

as $n \rightarrow \infty$ due to the one of our assumptions. Thus,

$$
u_{n}(x) \rightarrow u(x) \quad \text { in } \quad L^{2}\left(\mathbb{R}^{2}\right), \quad n \rightarrow \infty
$$

in the case of the dimension $d=2$ and $a=0$.

Then we proceed to the proof of the part b) of the theorem, when the dimension $d \geq 3$ and $a=0$. In this case, by means of the part b) of Theorem 1, equations (1.2) and (1.3) possess unique solutions $u(x)$ and $u_{n}(x), n \in \mathbb{N}$ respectively, belonging to $H^{1}\left(\mathbb{R}^{d}\right)$. Let us estimate the first term in the right side of (2.28) in the absolute value from above using (1.13) by

$$
\frac{\left\|f_{n}(x)-f(x)\right\|_{L^{1}\left(\mathbb{R}^{d}\right)}}{(2 \pi)^{\frac{d}{2}}|p|} \chi_{\{|p| \leq 1\}}, \quad d \geq 3,
$$

such that its $L^{2}\left(\mathbb{R}^{d}\right)$ norm can be bounded from above by

$$
C\left\|f_{n}(x)-f(x)\right\|_{L^{1}\left(\mathbb{R}^{d}\right)} \rightarrow 0, \quad n \rightarrow \infty
$$

due to the one of our assumptions. Therefore,

$$
u_{n}(x) \rightarrow u(x) \quad \text { in } \quad L^{2}\left(\mathbb{R}^{d}\right), \quad n \rightarrow \infty
$$

in the case of the dimension $d \geq 3$ and $a=0$.

Then we turn our attention to the case c) of the theorem, when the dimension $d=1$ and $a>0$. Hence, due to the result of the part c) of Theorem 1, equation (1.3) admits a unique solution $u_{n}(x) \in H^{1}(\mathbb{R}), n \in \mathbb{N}$. We have $f_{n}(x) \in L^{1}(\mathbb{R}), n \in \mathbb{N}$, such that $f_{n}(x) \rightarrow f(x)$ in $L^{1}(\mathbb{R})$ as $n \rightarrow \infty$ as a result of Lemma 6 below. By virtue of the limiting argument, similarly to the proof of (2.29) we arrive at the orthogonality conditions

$$
\left(f(x), \frac{e^{ \pm i a x}}{\sqrt{2 \pi}}\right)_{L^{2}(\mathbb{R})}=0 .
$$

Then by means of the result of the part c) of Theorem 1, equation (1.2) has a unique solution $u(x) \in H^{1}(\mathbb{R})$. By applying the standard Fourier transform (1.12) to both sides of (1.2) and (1.3), we arrive at

$$
\widehat{u}(p)=\frac{\widehat{f}(p)}{|p|-a}, \quad \widehat{u}_{n}(p)=\frac{\widehat{f}_{n}(p)}{|p|-a}, \quad n \in \mathbb{N} .
$$


This enables us to express $\widehat{u}_{n}(p)-\widehat{u}(p)$ as

$$
\begin{aligned}
& \frac{\widehat{f}_{n}(p)-\widehat{f}(p)}{p-a} \chi_{I_{\delta}^{+}}+\frac{\widehat{f}_{n}(p)-\widehat{f}(p)}{p-a} \chi_{I_{\delta}^{c+}}+ \\
& +\frac{\widehat{f}_{n}(p)-\widehat{f}(p)}{-p-a} \chi_{I_{\delta}^{-}}+\frac{\widehat{f}_{n}(p)-\widehat{f}(p)}{-p-a} \chi_{I_{\delta}^{c-}},
\end{aligned}
$$

where $I_{\delta}^{+}, I_{\delta}^{-}$are given by (2.24) and $I_{\delta}^{c+}, I_{\delta}^{c-}$ are defined in (2.25). Clearly, the second term in (2.37) can be bounded from above in the absolute value by $\frac{\left|\widehat{f}_{n}(p)-\widehat{f}(p)\right|}{\delta}$, such that

$$
\left\|\frac{\widehat{f}_{n}(p)-\widehat{f}(p)}{p-a} \chi_{I_{\delta}^{c+}}\right\|_{L^{2}(\mathbb{R})} \leq \frac{\left\|f_{n}(x)-f(x)\right\|_{L^{2}(\mathbb{R})}}{\delta} \rightarrow 0, \quad n \rightarrow \infty
$$

due to the one of our assumptions. Similarly, the last term in (2.37) can be estimated from above in the absolute value by $\frac{\left|\widehat{f}_{n}(p)-\widehat{f}(p)\right|}{\delta}$. Hence

$$
\left\|\frac{\widehat{f}_{n}(p)-\widehat{f}(p)}{-p-a} \chi_{I_{\delta}^{c-}}\right\|_{L^{2}(\mathbb{R})} \leq \frac{\left\|f_{n}(x)-f(x)\right\|_{L^{2}(\mathbb{R})}}{\delta} \rightarrow 0, \quad n \rightarrow \infty
$$

as assumed. Orthogonality conditions (2.35) and (1.10) yield

$$
\widehat{f}(a)=0, \quad \widehat{f}_{n}(a)=0, \quad n \in \mathbb{N},
$$

such that

$$
\widehat{f}(p)=\int_{a}^{p} \frac{d \widehat{f}(s)}{d s} d s, \quad \widehat{f}_{n}(p)=\int_{a}^{p} \frac{d \widehat{f}_{n}(s)}{d s} d s, \quad n \in \mathbb{N},
$$

which allows us to write the first term in (2.37) as

$$
\frac{\int_{a}^{p}\left[\frac{d \widehat{f}_{n}(s)}{d s}-\frac{d \widehat{f}(s)}{d s}\right] d s}{p-a} \chi_{I_{\delta}^{+}}
$$

Using (2.31), we estimate expression (2.38) from above in the absolute value by

$$
\frac{1}{\sqrt{2 \pi}}\left\|x f_{n}(x)-x f(x)\right\|_{L^{1}(\mathbb{R})} \chi_{I_{\delta}^{+}}
$$

Therefore, the $L^{2}(\mathbb{R})$ norm of (2.38) can be bounded from above by

$$
\sqrt{\frac{\delta}{\pi}}\left\|x f_{n}(x)-x f(x)\right\|_{L^{1}(\mathbb{R})} \rightarrow 0, \quad n \rightarrow \infty
$$


due to the one of our assumptions. Orthogonality relations (2.35) and (1.10) give us

$$
\widehat{f}(-a)=0, \quad \widehat{f}_{n}(-a)=0, \quad n \in \mathbb{N} .
$$

Hence, at the negative singularity

$$
\widehat{f}(p)=\int_{-a}^{p} \frac{d \widehat{f}(s)}{d s} d s, \quad \widehat{f}_{n}(p)=\int_{-a}^{p} \frac{d \widehat{f}_{n}(s)}{d s} d s, \quad n \in \mathbb{N} .
$$

This enables us to estimate the third term in (2.37) from above in the absolute value by

$$
\frac{1}{\sqrt{2 \pi}}\left\|x f_{n}(x)-x f(x)\right\|_{L^{1}(\mathbb{R})} \chi_{I_{\delta}^{-}} .
$$

Thus, its $L^{2}(\mathbb{R})$ norm can be bounded from above by

$$
\sqrt{\frac{\delta}{\pi}}\left\|x f_{n}(x)-x f(x)\right\|_{L^{1}(\mathbb{R})} \rightarrow 0, \quad n \rightarrow \infty
$$

by means of the one of our assumptions. This proves that in dimension $d=1$, when $a>0$ we have

$$
u_{n}(x) \rightarrow u(x) \quad \text { in } \quad L^{2}(\mathbb{R}), \quad n \rightarrow \infty .
$$

Let us conclude the proof of the theorem with addressing the case d) when the dimension $d \geq 2$ and $a>0$. Then under the given assumptions, by means of the part d) of Theorem 1, equation (1.3) admits a unique solution $u_{n}(x) \in H^{1}\left(\mathbb{R}^{d}\right), n \in \mathbb{N}$. An easy limiting argument similar to the proof of (2.35) yields

$$
\left(f(x), \frac{e^{i p x}}{(2 \pi)^{\frac{d}{2}}}\right)_{L^{2}\left(\mathbb{R}^{d}\right)}=0, \quad p \in S_{a}^{d} .
$$

Then by virtue of the part d) of Theorem 1, equation (1.2) has a unique solution $u(x) \in H^{1}\left(\mathbb{R}^{d}\right)$. Using the Fourier transform (1.12), we easily obtain

$$
\widehat{u}_{n}(p)-\widehat{u}(p)=\frac{\widehat{f}_{n}(p)-\widehat{f}(p)}{|p|-a} \chi_{A_{\delta}}+\frac{\widehat{f}_{n}(p)-\widehat{f}(p)}{|p|-a} \chi_{A_{\delta}^{c}},
$$

where the set $A_{\delta}$ is defined in (2.26). Clearly, the second term in the right side of (2.40) can be bounded from above in the absolute value by $\frac{\left|\widehat{f}_{n}(p)-\widehat{f}(p)\right|}{\delta}$. Thus,

$$
\left\|\frac{\widehat{f}_{n}(p)-\widehat{f}(p)}{|p|-a} \chi_{A_{\delta}^{c}}\right\|_{L^{2}\left(\mathbb{R}^{d}\right)} \leq \frac{\left\|f_{n}(x)-f(x)\right\|_{L^{2}\left(\mathbb{R}^{d}\right)}}{\delta} \rightarrow 0
$$

as $n \rightarrow \infty$ due to the one of our assumptions. Orthogonality conditions (2.39) and (1.11) give us

$$
\widehat{f}(a, \sigma)=0, \quad \widehat{f}_{n}(a, \sigma)=0, \quad n \in \mathbb{N},
$$


such that

$$
\widehat{f}(p)=\int_{a}^{|p|} \frac{\partial \widehat{f}(s, \sigma)}{\partial s} d s, \quad \widehat{f}_{n}(p)=\int_{a}^{|p|} \frac{\partial \widehat{f}_{n}(s, \sigma)}{\partial s} d s, \quad n \in \mathbb{N} .
$$

By virtue of the definition of the Fourier transform (1.12), similarly to inequalities (2.31) and (2.34) in lower dimensions, we easily derive

$$
\left|\frac{\partial \widehat{f}_{n}(p)}{\partial|p|}-\frac{\partial \widehat{f}(p)}{\partial|p|}\right| \leq \frac{1}{(2 \pi)^{\frac{d}{2}}}\left\|x f_{n}(x)-x f(x)\right\|_{L^{1}\left(\mathbb{R}^{d}\right)} .
$$

Let us estimate the first term in the right side of (2.40) from above in the absolute value by

$$
\frac{1}{(2 \pi)^{\frac{d}{2}}}\left\|x f_{n}(x)-x f(x)\right\|_{L^{1}\left(\mathbb{R}^{d}\right)} \chi_{A_{\delta}} .
$$

Therefore,

$$
\left\|\frac{\widehat{f_{n}}(p)-\widehat{f}(p)}{|p|-a} \chi_{A_{\delta}}\right\|_{L^{2}\left(\mathbb{R}^{d}\right)} \leq C\left\|x f_{n}(x)-x f(x)\right\|_{L^{1}\left(\mathbb{R}^{d}\right)} \rightarrow 0, \quad n \rightarrow \infty
$$

as assumed. This implies that in dimensions $d \geq 2$, when $a>0$ we have

$$
u_{n}(x) \rightarrow u(x) \quad \text { in } \quad L^{2}\left(\mathbb{R}^{d}\right)
$$

as $n \rightarrow \infty$.

\section{Solvability in the sense of sequences with a scalar potential}

Proof of Theorem 4. Note that the case a) of the theorem is the result of Lemma 3.3 of [29]. Then we proceed to proving the case of $a>0$.

Let us note that it is sufficient to solve equation (1.14) in $L^{2}\left(\mathbb{R}^{3}\right)$, since its square integrable solution will belong to $H^{1}\left(\mathbb{R}^{3}\right)$ as well. Indeed, it can be easily verified that

$$
\|\sqrt{-\Delta+V(x)} u\|_{L^{2}\left(\mathbb{R}^{3}\right)}^{2}=\|\nabla u\|_{L^{2}\left(\mathbb{R}^{3}\right)}^{2}+\int_{\mathbb{R}^{3}} V(x)|u(x)|^{2} d x
$$

where $u(x)$ is a square integrable solutions of (1.14), the scalar potential $V(x)$ is bounded due to Assumption 3 and $f(x) \in L^{2}\left(\mathbb{R}^{3}\right)$ as assumed. Then by means of indentity (3.41) we have $\nabla u(x) \in L^{2}\left(\mathbb{R}^{3}\right)$ and therefore, $u(x) \in H^{1}\left(\mathbb{R}^{3}\right)$.

To address the uniqueness of solutions of our problem, let us suppose that there exist both $u_{1}(x)$ and $u_{2}(x)$ which are square integrable in $\mathbb{R}^{3}$ and satisfy (1.14). Then their difference $w(x):=u_{1}(x)-u_{2}(x) \in L^{2}\left(\mathbb{R}^{3}\right)$ is a solution of the equation

$$
\sqrt{-\Delta+V(x)} w=a w .
$$


Since the operator $\sqrt{-\Delta+V(x)}$ has no nontrivial $L^{2}\left(\mathbb{R}^{3}\right)$ eigenfunctions as discussed above, $w(x)$ vanishes a.e. in $\mathbb{R}^{3}$.

We apply the generalized Fourier transform (1.20) with respect to the functions of the continuous spectrum of the Schrödinger operator to both sides of equation (1.14), which gives us

$$
\tilde{u}(k)=\frac{\tilde{f}(k)}{|k|-a} .
$$

Let us introduce the spherical layer in the space of three dimensions as

$$
B_{\delta}:=\left\{k \in \mathbb{R}^{3}|a-\delta \leq| k \mid \leq a+\delta\right\}, 0<\delta<a .
$$

This allows us to express

$$
\tilde{u}(k)=\frac{\tilde{f}(k)}{|k|-a} \chi_{B_{\delta}}+\frac{\tilde{f}(k)}{|k|-a} \chi_{B_{\delta}^{c}}
$$

The second term in the right side of (3.43) can be easily estimated from above in the absolute value by

$$
\frac{|\tilde{f}(k)|}{\delta} \in L^{2}\left(\mathbb{R}^{3}\right)
$$

since $f(x)$ is square integrable as assumed. Let us use the representation

$$
\tilde{f}(k)=\tilde{f}(a, \sigma)+\int_{a}^{|k|} \frac{\partial \tilde{f}(s, \sigma)}{\partial s} d s .
$$

Hence, the first term in the right side of (3.43) can be written as

$$
\frac{\tilde{f}(a, \sigma)}{|k|-a} \chi_{B_{\delta}}+\frac{\int_{a}^{|k|} \frac{\partial \tilde{f}(s, \sigma)}{\partial s} d s}{|k|-a} \chi_{B_{\delta}} .
$$

The second term in sum (3.44) can be easily bounded above in the absolute value by

$$
\left\|\nabla_{k} \tilde{f}(k)\right\|_{L^{\infty}\left(\mathbb{R}^{3}\right)} \chi_{B_{\delta}} \in L^{2}\left(\mathbb{R}^{3}\right) .
$$

Note that under our assumptions $\nabla_{k} \tilde{f}(k) \in L^{\infty}\left(\mathbb{R}^{3}\right)$ by virtue of Lemma 2.4 of [19]. The first term in (3.44) belongs to $L^{2}\left(\mathbb{R}^{3}\right)$ if and only if $\tilde{f}(a, \sigma)$ vanishes, which gives orthogonality condition (1.22).

Then we proceed to the proof of our last main proposition concerning the solvability in the sense of sequences.

Proof of Theorem 5. First of all, let us show that if $u(x)$ and $u_{n}(x), n \in \mathbb{N}$ are the unique $H^{1}\left(\mathbb{R}^{3}\right)$ solutions of (1.14) and (1.15) respectively and $u_{n}(x) \rightarrow u(x)$ in $L^{2}\left(\mathbb{R}^{3}\right)$ as $n \rightarrow \infty$, then we have $u_{n}(x) \rightarrow u(x)$ in $H^{1}\left(\mathbb{R}^{3}\right)$ as $n \rightarrow \infty$ as well. 
Indeed, from (1.14) and (1.15) we easily deduce that for $a \geq 0$

$$
\sqrt{-\Delta+V(x)}\left(u_{n}(x)-u(x)\right)=a\left(u_{n}(x)-u(x)\right)+\left(f_{n}(x)-f(x)\right), \quad n \in \mathbb{N} .
$$

Hence

$$
\begin{gathered}
\left\|\sqrt{-\Delta+V(x)}\left(u_{n}(x)-u(x)\right)\right\|_{L^{2}\left(\mathbb{R}^{3}\right)} \leq a\left\|u_{n}(x)-u(x)\right\|_{L^{2}\left(\mathbb{R}^{3}\right)}+ \\
+\left\|f_{n}(x)-f(x)\right\|_{L^{2}\left(\mathbb{R}^{3}\right)} \rightarrow 0, \quad n \rightarrow \infty
\end{gathered}
$$

as assumed. Let us express

$$
\begin{gathered}
\left\|\sqrt{-\Delta+V(x)}\left(u_{n}(x)-u(x)\right)\right\|_{L^{2}\left(\mathbb{R}^{3}\right)}^{2}=\left\|\nabla\left(u_{n}(x)-u(x)\right)\right\|_{L^{2}\left(\mathbb{R}^{3}\right)}^{2}+ \\
+\int_{\mathbb{R}^{3}} V(x)\left|u_{n}(x)-u(x)\right|^{2} d x,
\end{gathered}
$$

where the scalar potential $V(x)$ is bounded due to Assumption 3. Hence, in the identity above the left side and the second term in the right side tend to zero as $n \rightarrow \infty$. This implies that $\nabla u_{n}(x) \rightarrow \nabla u(x)$ in $L^{2}\left(\mathbb{R}^{3}\right)$ as $n \rightarrow \infty$. Therefore, $u_{n}(x) \rightarrow u(x)$ in $H^{1}\left(\mathbb{R}^{3}\right)$ as $n \rightarrow \infty$ as well.

In the case a) equations (1.14) and (1.15) admit unique solutions $u(x), u_{n}(x) \in$ $H^{1}\left(\mathbb{R}^{3}\right), n \in \mathbb{N}$ respectively by means of the part a) of Theorem 4 above. By applying the generalized Fourier transform (1.20) to both sides of problems (1.14) and (1.15), we arrive at

$$
\tilde{u}(k)=\frac{\tilde{f}(k)}{|k|}, \quad \tilde{u}_{n}(k)=\frac{\tilde{f}_{n}(k)}{|k|}, \quad n \in \mathbb{N} .
$$

Hence

$$
\tilde{u}_{n}(k)-\tilde{u}(k)=\frac{\tilde{f}_{n}(k)-\tilde{f}(k)}{|k|} \chi_{\{|k| \leq 1\}}+\frac{\tilde{f}_{n}(k)-\tilde{f}(k)}{|k|} \chi_{\{|k|>1\}} .
$$

Clearly, the second term in the right side of (3.45) can be easily bounded from above in the absolute value by $\left|\tilde{f}_{n}(k)-\tilde{f}(k)\right|$, such that

$$
\left\|\frac{\tilde{f}_{n}(k)-\tilde{f}(k)}{|k|} \chi_{\{|k|>1\}}\right\|_{L^{2}\left(\mathbb{R}^{3}\right)} \leq\left\|f_{n}(x)-f(x)\right\|_{L^{2}\left(\mathbb{R}^{3}\right)} \rightarrow 0, \quad n \rightarrow \infty
$$

due to the one of our assumptions. Let us estimate the first term in the right side of (3.45) from above in the absolute value using (1.21) by

$$
\frac{1}{(2 \pi)^{\frac{3}{2}}} \frac{1}{1-I(V)}\left\|f_{n}(x)-f(x)\right\|_{L^{1}\left(\mathbb{R}^{3}\right)} \frac{\chi_{\{|k| \leq 1\}}}{|k|} .
$$


Evidently, this implies that

$$
\left\|\frac{\tilde{f}_{n}(k)-\tilde{f}(k)}{|k|} \chi_{\{|k| \leq 1\}}\right\|_{L^{2}\left(\mathbb{R}^{3}\right)} \leq \frac{1}{\sqrt{2} \pi} \frac{1}{1-I(V)}\left\|f_{n}(x)-f(x)\right\|_{L^{1}\left(\mathbb{R}^{3}\right)} \rightarrow 0
$$

as $n \rightarrow \infty$ by means of the one of our assumptions. Therefore, $u_{n}(x) \rightarrow u(x)$ in $L^{2}\left(\mathbb{R}^{3}\right)$ as $n \rightarrow \infty$ in the case when the parameter $a=0$.

Then we turn our attention to the proof of the part b) of the theorem. By means of the result the part $b$ ) of Theorem 4 , each equation (1.15) admits a unique solution $u_{n}(x) \in H^{1}\left(\mathbb{R}^{3}\right), \quad n \in \mathbb{N}$. Then for $k \in S_{a}^{3}$ a.e. via (1.21), we have

$$
\begin{aligned}
& \left|\left(f(x), \varphi_{k}(x)\right)_{L^{2}\left(\mathbb{R}^{3}\right)}\right|=\left|\left(f(x)-f_{n}(x), \varphi_{k}(x)\right)_{L^{2}\left(\mathbb{R}^{3}\right)}\right| \leq \\
& \leq \frac{1}{(2 \pi)^{\frac{3}{2}}} \frac{1}{1-I(V)}\left\|f_{n}(x)-f(x)\right\|_{L^{1}\left(\mathbb{R}^{3}\right)} \rightarrow 0, \quad n \rightarrow \infty
\end{aligned}
$$

by virtue of our assumptions along with Lemma 6 below. Therefore,

$$
\left(f(x), \varphi_{k}(x)\right)_{L^{2}\left(\mathbb{R}^{3}\right)}=0, \quad k \in S_{a}^{3}
$$

holds. Then by virtue of the result the part b) of Theorem 4, equation (1.14) possesses a unique solution $u(x) \in H^{1}\left(\mathbb{R}^{3}\right)$. Let us apply the generalized Fourier transform (1.20) to both sides of equations (1.14) and (1.15). Thus, we arrive at

$$
\tilde{u}_{n}(k)-\tilde{u}(k)=\frac{\tilde{f}_{n}(k)-\tilde{f}(k)}{|k|-a} \chi_{B_{\delta}}+\frac{\tilde{f}_{n}(k)-\tilde{f}(k)}{|k|-a} \chi_{B_{\delta}^{c}}
$$

with $B_{\delta}$ defined in (3.42). Clearly, the second term in the right side of (3.47) can be bounded from above in the absolute value by $\frac{\left|\tilde{f}_{n}(k)-\tilde{f}(k)\right|}{\delta}$, such that

$$
\left\|\frac{\tilde{f}_{n}(k)-\tilde{f}(k)}{|k|-a} \chi_{B_{\delta}^{c}}\right\|_{L^{2}\left(\mathbb{R}^{3}\right)} \leq \frac{\left\|f_{n}(x)-f(x)\right\|_{L^{2}\left(\mathbb{R}^{3}\right)}}{\delta} \rightarrow 0, \quad n \rightarrow \infty
$$

according to the one of our assumptions. By means of orthogonality conditions (1.23) and (3.46), we have

$$
\tilde{f}(a, \sigma)=0, \quad \tilde{f}_{n}(a, \sigma)=0, \quad n \in \mathbb{N} .
$$

This yields the representations

$$
\tilde{f}(k)=\int_{a}^{|k|} \frac{\partial \tilde{f}(s, \sigma)}{\partial s} d s, \quad \tilde{f}_{n}(k)=\int_{a}^{|k|} \frac{\partial \tilde{f}_{n}(s, \sigma)}{\partial s} d s, \quad n \in \mathbb{N},
$$

such that the first term in the right side of (3.47) can be written as

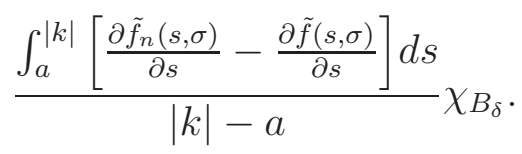


Expression (3.48) can be easily bounded from above in the absolute value by

$$
\left\|\nabla_{k}\left[\tilde{f}_{n}(k)-\tilde{f}(k)\right]\right\|_{L^{\infty}\left(\mathbb{R}^{3}\right)} \chi_{B_{\delta}} .
$$

Hence the $L^{2}\left(\mathbb{R}^{3}\right)$ norm of (3.48) can be estimated from above by

$$
C\left\|\nabla_{k}\left[\tilde{f}_{n}(k)-\tilde{f}(k)\right]\right\|_{L^{\infty}\left(\mathbb{R}^{3}\right)} \rightarrow 0, \quad n \rightarrow \infty
$$

by means of Lemma 3.4 of [28] under our assumptions. This proves that $u_{n}(x) \rightarrow$ $u(x)$ in $L^{2}\left(\mathbb{R}^{3}\right)$ as $n \rightarrow \infty$ when $a>0$.

\section{Auxiliary results}

The following elementary lemma is used when establishing the solvability in the sense of sequences in the theorems above, with and without a scalar potential.

Lemma 6. a) Let $f(x) \in L^{2}\left(\mathbb{R}^{d}\right), d \in \mathbb{N}$ and $x f(x) \in L^{1}\left(\mathbb{R}^{d}\right)$. Then $f(x) \in$ $L^{1}\left(\mathbb{R}^{d}\right)$.

b) Let $n \in \mathbb{N}, f_{n}(x) \in L^{2}\left(\mathbb{R}^{d}\right), d \in \mathbb{N}$, such that $f_{n}(x) \rightarrow f(x)$ in $L^{2}\left(\mathbb{R}^{d}\right)$ as $n \rightarrow \infty$. Let $x f_{n}(x) \in L^{1}\left(\mathbb{R}^{d}\right)$, such that $x f_{n}(x) \rightarrow x f(x)$ in $L^{1}\left(\mathbb{R}^{d}\right)$ as $n \rightarrow \infty$. Then $f_{n}(x) \rightarrow f(x)$ in $L^{1}\left(\mathbb{R}^{d}\right)$ as $n \rightarrow \infty$.

Proof. To prove the part a), we express the norm $\|f(x)\|_{L^{1}\left(\mathbb{R}^{d}\right)}$ as

$$
\int_{|x| \leq 1}|f(x)| d x+\int_{|x|>1}|f(x)| d x .
$$

By applying the Schwarz inequality, this can be bounded from above by

$$
\begin{gathered}
\sqrt{\int_{|x| \leq 1}|f(x)|^{2} d x} \sqrt{\int_{|x| \leq 1} 1 d x}+\int_{|x|>1}|x||f(x)| d x \leq \\
\leq\|f(x)\|_{L^{2}\left(\mathbb{R}^{d}\right)} \sqrt{\left|B^{d}\right|}+\|x f(x)\|_{L^{1}\left(\mathbb{R}^{d}\right)}<\infty
\end{gathered}
$$

due to our assumptions.

To show that the part b) holds, we trivially estimate the norm using the Schwarz inequality as

$$
\begin{aligned}
& \left\|f_{n}(x)-f(x)\right\|_{L^{1}\left(\mathbb{R}^{d}\right)} \leq \\
& \leq \sqrt{\int_{|x| \leq 1}\left|f_{n}(x)-f(x)\right|^{2} d x} \sqrt{\int_{|x| \leq 1} 1 d x}+\int_{|x|>1}|x|\left|f_{n}(x)-f(x)\right| d x \leq \\
& \leq\left\|f_{n}(x)-f(x)\right\|_{L^{2}\left(\mathbb{R}^{d}\right)} \sqrt{\left|B^{d}\right|}+\left\|x f_{n}(x)-x f(x)\right\|_{L^{1}\left(\mathbb{R}^{d}\right)} \rightarrow 0, n \rightarrow \infty
\end{aligned}
$$

by means of our assumptions. 


\section{Acknowledgements}

The second author was partially supported by the Ministry of Education and Science of the Russian Federation (the agreement number 02.a03.21.0008).

\section{References}

[1] C. Amrouche, V. Girault, J. Giroire, Dirichlet and Neumann exterior problems for the n-dimensional Laplace operator. An approach in weighted Sobolev spaces, J. Math, Pures Appl., 76 (1997), 55-81.

[2] C. Amrouche, F. Bonzom, Mixed exterior Laplace's problem, J. Math. Anal. Appl., 338 (2008), 124-140.

[3] P. Bolley, T.L. Pham, Propriété d'indice en théorie Holderienne pour des opérateurs différentiels elliptiques dans $R^{n}$, J. Math. Pures Appl., 72 (1993), 105-119.

[4] P. Bolley, T.L. Pham, Propriété d'indice en théorie Hölderienne pour le problème extérieur de Dirichlet, Comm. Partial Differential Equations, 26 (2001), No. 1-2, 315-334.

[5] N. Benkirane, Propriété d'indice en théorie Holderienne pour des opérateurs elliptiques dans $R^{n}$, CRAS, 307, Série I (1988), 577-580.

[6] H.L. Cycon, R.G. Froese, W. Kirsch, B. Simon, Schrödinger Operators with Application to Quantum Mechanics and Global Geometry, Springer-Verlag, Berlin (1987).

[7] A. Ducrot, M. Marion, V. Volpert, Systemes de réaction-diffusion sans propriété de Fredholm, CRAS, 340 (2005), 659-664.

[8] A. Ducrot, M. Marion, V. Volpert, Reaction-diffusion problems with non Fredholm operators, Advances Diff. Equations, 13 (2008), No. 11-12, 11511192.

[9] M. Goldberg, W. Schlag, A limiting absorption principle for the threedimensional Schrödinger equation with $L^{p}$ potentials, Int. Math. Res. Not., (2004), No. 75, 4049-4071.

[10] P.D. Hislop, I.M. Sigal, Introduction to Spectral Theory with Applications to Schrödinger Operators. Springer, 1996.

[11] B.L.G. Jonsson, M. Merkli, I.M. Sigal, F. Ting, Applied Analysis, In preparation. 
[12] T. Kato, Wave operators and similarity for some non-selfadjoint operators, Math. Ann., 162 (1965/1966), 258-279.

[13] E. Lieb, M. Loss, Analysis. Graduate Studies in Mathematics, 14, American Mathematical Society, Providence (1997).

[14] R. Metzler, J. Klafter, The random walk's guide to anomalous diffusion: a fractional dynamics approach, Phys. Rep., 339, (2000), 1-77.

[15] M. Reed, B. Simon, Methods of Modern Mathematical Physics, III: Scattering Theory, Academic Press (1979).

[16] I. Rodnianski, W. Schlag, Time decay for solutions of Schrödinger equations with rough and time-dependent potentials, Invent. Math., 155 (2004), No. 3, 451-513.

[17] V. Volpert. Elliptic partial differential equations. Volume 1. Fredholm theory of elliptic problems in unbounded domains. Birkhauser, 2011.

[18] V. Volpert, B. Kazmierczak, M. Massot, Z.Peradzynski, Solvability conditions for elliptic problems with non-Fredholm operators, Appl.Math., 29 (2002), No. 2, 219-238.

[19] V. Vougalter, V. Volpert, Solvability conditions for some non-Fredholm operators, Proc. Edinb. Math. Soc. (2), 54 (2011), No.1, 249-271

[20] V. Vougalter, V. Volpert. On the solvability conditions for some non Fredholm operators, Int. J. Pure Appl. Math., 60 (2010), No. 2, 169-191.

[21] V. Vougalter, V. Volpert. On the solvability conditions for the diffusion equation with convection terms, Commun. Pure Appl. Anal., 11 (2012), No. 1, 365-373.

[22] V. Vougalter, V. Volpert. Solvability relations for some non Fredholm operators, Int. Electron. J. Pure Appl. Math., 2 (2010), No. 1, 75-83.

[23] V. Volpert, V. Vougalter. On the solvability conditions for a linearized CahnHilliard equation, Rend. Istit. Mat. Univ. Trieste, 43 (2011), 1-9.

[24] V. Vougalter. Sharp semiclassical bounds for the moments of eigenvalues for some Schrödinger type operators with unbounded potentials, Math. Model. Nat. Phenom., 8 (2013), No. 1, 237-245.

[25] V. Vougalter, V. Volpert. Solvability conditions for a linearized Cahn-Hilliard equation of sixth order, Math. Model. Nat. Phenom., 7 (2012), No. 2, 146-154. 
[26] V. Vougalter, V. Volpert. Solvability conditions for some linear and nonlinear non-Fredholm elliptic problems, Anal. Math. Phys., 2 (2012), No.4, 473-496.

[27] V. Vougalter, V. Volpert. On the solvability in the sense of sequences for some non-Fredholm operators, Dyn. Partial Differ. Equ., 11 (2014), No.2, 109-124.

[28] V. Volpert, V. Vougalter. Solvability in the sense of sequences to some nonFredholm operators, Electron. J. Differential Equations, 2013, No. 160, 16pp.

[29] V. Vougalter, V. Volpert. Existence of stationary solutions for some integrodifferential equations with superdiffusion, to appear in Rend. Semin. Mat. Univ. Padova. 